\title{
TALEN-edited HPV16/18 E6/E7 Plasmid
}

National Cancer Institute

\section{Source}

National Cancer Institute. TALEN-edited HPV16/18 E6/E7 Plasmid. NCI Thesaurus. Code C148186.

A transcription activator-like effector nuclease (TALEN)-edited plasmid targeting human papillomavirus (HPV) types 16 (HPV16) and 18 (HPV18) epitopes E6 and E7, with potential antineoplastic activity. Upon administration of TALEN-edited HPV16/18 E6/E7, the TALEN targets and binds to specific sites on genomic HPV16 and 18 E6 and E7, and cleaves the DNA sequences encoding E6 and E7. This causes double-strand DNA (dsDNA) breaks, which prevents the transcription and translation of E6 and E7. In addition, inhibition of HPV16/18 E6/E7 increases the expression of tumor suppressor genes, such as p53 and retinoblastoma 1 (RB1). Altogether, TALEN-based HPV editing increases apoptosis and inhibits tumor cell proliferation of HPV-driven cancer cells. HPV16/18 E6 and E7 are oncogenes vital to viral function and carcinogenesis. 